\title{
TOWARDS SITE 2000 DEVELOPMENTS IN AUTOMATION AND ROBOTICS IN CONSTRUCTION
}

\author{
Dr David A. Bradley and Derek W. Seward, \\ Engineering Department, \\ Lancaster University, Bailrigg, Lancaster LA1 4YR, United Kingdom. \\ Dr Fikry K. Garas, \\ Taylor Woodrow, \\ Taywood House, 345 Ruislip Road, Southall, Middlesex UB1 2QX, \\ United Kingdom.
}

\begin{abstract}
The current state of robotic technology is briefly reviewed with a view to establishing what developments need to take place to enable the fully integrated site of the future. A scenario is presented depicting how such a project could be organised - from tender stage to completion and implications are drawn from this. It is concluded that such a scenario could become reality using largely current technology, but that the real barriers to implementation involve issues of management, organisation and attitude. A proposal for an international test-bed site is made.
\end{abstract}

\section{INTRODUCTION}

The purpose of this paper is to look ahead to the year 2000 and beyond and to speculate as to whether the current early developments in construction automation and robotics will flourish and grow or whether they will wither and die. The current state is one of fragmented and piecemeal development with new technology firmly established in some areas but other areas left untouched. Table 1 illustrates the current state of development of new technology in the construction industry.

The explosion in cheap computing power that has taken place has resulted in the items listed in the first category being widely used for reasons of both economics and product quality. It would be expected that other items should gradually move-up through the categories as the technology advances. However, there is growing awareness in the construction robotics research community that no significant leap forward will be made until the items listed under future developments are adequately addressed.

What follows is the presentation of a scenario for SITE 2000 to illustrate how the full integration of new technology might influence a typical medium sized construction project such as a hospital or large office complex. 


\section{TABLE 1 - Current status of new technologies in construction ${ }^{1}$}

Firmly established

Existing but not

widely used

Total-station surveying

Computer-aided design

Computer-aided drafting

Computer scheduling, quantities and bills of quantities

Automated and robotic fabrication of steelwork

Automatic bending of concrete reinforcement

Laser guidance of certain types of plant

Ground penetrating radar

Flexible manufacturing of reinforced concrete components

Remote and telecheric handling devices

Inspection devices for inaccessible locations

Painting and fireproofing robots

Concrete placement

Floor finishing robots

Remotely controlled excavating plant

\section{Currently being researched or developed}

Satellite and other navigation systems

Robotic cranes for erection of components

Intelligent, autonomous robotic excavators

Multi-purpose inspection and maintenance devices

Implications for the design process

\section{Future developments}

On and off-site integration of new technologies

Task planning

Reform of contractual and management practices

Safety systems

Flexible modularisation of components

Information technology and site information systems

\section{A SCENARIO FOR SITE 2000}

\section{STAGE 1 - THE TENDER}

A leading design-and build consortium is approached by a client to provide a quote for a large hospital complex which is to be built on a site on the outskirts of a large European city. The consortiums computer system receives from the client:-

- An outline specification.

- A 3-D model of the site including the results of a deep-penetrating radar survey of the sub-soil,and details of local services and highways.

- Contractual details, timetable and details of contact personnel.

- Recommendations of an earlier feasibility study. 
The consortium immediately invokes its powerful preliminary design and visualisation software. A knowledge-based computer system works alongside the project architect to check compliance with the specification and to provide advice as requested. This allows the architect more time to explore the full creative potential of the project. Advanced graphics terminals allow rapid 3-D visualisations to be created. The architect has access to an extensive library of standard modules as well as the ability to rapidly create new modules either by combining primitive shapes or from a sketchpad linked to the terminal. The new models can inherit any of the characteristics of existing modules. As the new building is synthesized the knowledge base applies heuristic procedures to provide an instant cost estimate. Unusual structural problems are highlighted for closer investigation by the engineer.

Once a scheme has been developed, the architect is assisted in producing promotional material including a walk-through graphical computer simulation. The data is automatically produced for the manufacture of a 3-D plastic model which is made by laser forming.

\section{STAGE 2 - SitE PREPARATION}

After winning the contract the consortium continues with the detail design at the same time as preparing the site. A semi-automatic machine is brought on site by the fencing subcontractor and within a few days has completed the $3 \mathrm{~km}$ of perimeter fence. Laser bar-code readers are positioned at each site entrance and exit to monitor the movement of all materials, plant and personnel. Little space is required for the storage compound because the site will operate on a just-in-time philosophy. Also many more components will be factory produced. Likewise little space is required for the site huts as relatively few site staff will be employed. The air-conditioned mobile site control, command and communication unit lies at the hub of a small collection of mobile offices.

Two satellite communication beacons are erected at the perimeter of the site and their global coordinates carefully measured. The position of all plant on site will then be measured, by satellite, relative to these beacons to within an accuracy of $1 \mathrm{~mm}$. Where greater accuracy is required local references will be provided.

Heavy robotic plant then arrives on site to carry out the bulk earth-moving and compacting. The repetitive, incremental and cyclic nature of their work responds well to automation, and the 24 hour continuous working means that the work is complete in about a third of the normal time. Each machine contains sensors and enough local intelligence to optimise its performance in all types of ground. The overall control and coordination is however provided by the central control unit. The safety of such powerful machines is ensured by failsafe cut-outs if:-

- Machines get too close to each other.

- A machine gets too close to one of the safety transmitters worn by all site personnel.

- A machine gets within a given distance of the perimeter fence.

\section{STAGE 3 - THE SUB-STRUCTURE}

Once the bulk earth-moving is complete a small number of robot trenchers, hydraulic excavators, pipelayers and piling rigs are brought onto site. Both pipe laying and the construction of strip foundations are transformed from the previous discrete events to continuous 24-hour processes. No human workers are required to enter the trenches, thus removing one of the prime 
causes of death on construction sites. The global planning of the operation is carried out by the central control unit based on data supplied to it by the CAD software used in the design office.

Each work-cell of machines has enough autonomy to optimise its performance. For example, the pipe-layer pressure-tests each length of pipe as it goes along so that backfilling can take place immediately. Concrete foundations are laid continuously as the trench advances and before the sub-soil can be adversely affected by we ther. Robot excavation is more accurate than when done manually and so their is considerable saving from the absence of overdig. The site navigation and positioning system completely removes the need for setting out. The driving of piles is speeded up considerably and made more accurate by the auto-positioning facility.

\section{STAGE 4 - THE SUPERSTRUCTURE}

The traffic through the site gates increases rapidly as the materials for the construction of the superstructure arrive. Each delivery is checked against the design specification by the barcode readers and directed to the appropriate part of the site. Steel is erected by work-cells consisting of tower cranes and small mobile manipulators. Delivery vehicles are unloaded in designated delivery bays adjacent to the appropriate work-cell. The tower cranes are of course capable of managing their own collision avoidance.

Robot bricklayers operate continuously under special weather protection, but much of the brickwork arrives on site in the form of factory made panels, which are swung into position by special handling robots.

Throughout, the site information systems monitor the on-site flow of men and materials providing information on plant utilisation and deployment and enabling a rapid re-scheduling of resources in the face of unpredicatable factors such as changes in weather conditions. At the same time, the system feeds information to the contractors and suppliers, generates orders for materials and schedules their delivery on-site. The use of distributed data collection systems provides a direct input to quality control functions, both on site and at suppliers.

\section{STAGE 5 - FINISHES}

With conventional building techniques the application of finishes can often be the most time consuming stage of the construction process. Many of the factory made components which arrive at SITE 2000 are already finished to a high standard and require little or no further work. However those tasks which still remain to be completed are ideally suited to robots. Tedious tasks such as painting, applying fire-proof coatings, laying floor screeds and plastering are all carried out by specialist robots working continuously for 24 hours a day. Long and slender roof-tiling robots pick up tiles from a pallet and place them in position on the roof in accordance with the plans.

On completion, cleaning robots sweep and polish their way through the building before the final hand-over to the client.

\section{STAGE 6 - INSPECTION, MAINTENANCE AND REPAIR}

Throughout the life of the building a dedicated robot monitors its condition and carries out routine cleaning and maintenance tasks. Once a month the windows are cleaned and twice a year a structural survey of the exterior of the building is carried out. Any cracks or other defects 
such as loose tiles or defective sealant are investigated and reported to the building manager. Bolt-on modules are available to enable the robot to carry out particular maintenance and repair tasks.

Many of the information systems installed to monitor the construction process will now be transfered to monitoring the condition of the structure. Data colected by these systems during construction will form the basis of a reference data base against which the performance of the structure is monitored. Additional information systems for environmental control, security, safety and internal communications will be integrated with the construction data system as part of the structure and environment of the 'intelligent building' that results.

\section{IMPLICATIONS OF THE SITE 2000 SCENARIO}

If anything like the above scenario is ever to be realised then it is clear that not only is there a need for technological development but also that many attitudes must change in the traditionally conservative construction industry. The following implications can be drawn from the scenario.

- The golden rule when designing automated and robotic systems is to simplify the world. In other words do not attempt to solve a more difficult problem than necessary. This means that a robotic site of the future must be a more ordered place than the sometimes confused environment of the conventional site.

- It follows from the above that the piecemeal development of robotic systems must inevitable be severely limited in scope and function, and that if progress beyond a very limited threshold is to be achieved, a radical integration must be achieved.

- The extra cost of adding sensors, intelligence and communication to existing plant could be insignificant compared to the savings that can be achieved from greater efficiency. However the development of more specialist robots will only be economic when continuity of work can be guaranteed for them.

- The vital integration between the design and construction processes suggests that package deal type contracts will have many advantages. The legal implications of errors contained in information transfers will be minimised. Eventually this could lead to the breakdown of the traditional binary divide between designer and contractor.

- The specialist nature of the different technologies, high capital cost and dramatically increased performance points to the possibility that sub-contractors will specialise in operating one particular type of robot. However there must of course be total compatibility between the different systems and so a common protocol is now required.

- Greater use will be made of factory produced modular components. There is in the U.K. a high degree of disillusionment with modular or 'system' building as a result of poor quality projects completed in the sixties and early seventies. Many high-rise and low density buildings suffered from poor detailing and lax site quality control, resulting in leaking and rapidly deteriorating structures. Nevertheless, the benefits to the robotic site of the future are so great that the effort must be made to restore confidence in factory produced systems. New techniques of flexible manufacture will mean that interesting and individual components will be able to be produced. Automated and robotic assembly 
methods on site will mean that the vital quality of site connections will be assured.

- A range of standard connection details which facilitate robotic assembly techniques must be developed for all materials.. These would ensure the positive location of all components. Standardisation would ensure that components from different suppliers would fit together, and the designs would be optimised by experimental testing.

- The degree of automation depicted in this scenario is clearly most appropriate to large green-field construction projects. However there will undoubtedly be spin-off to smaller and more congested sites where the benefits of full integration cannot be realised.

- Information technology has an important role to play in the collection, managment and utilisation of data. By directly linking the design office with the site and with the work in progress there is the opportunity for significant improvements in areas such as quality, plant utilisation and safety. In addition, the automatic generation of orders, such as for ready mix concrete, would be a major requirement of the introduction of just-in-time operation on-site.

- The presence and use of robots and automated systems will reduce the levels of risk onsite by removing the need for site personnel to enter hazardous environments.

\section{CONCLUSIONS}

It can be concluded from the above that the achievement of an automated robotic construction site is not primarily technology limited. Clearly significant technical development is required but this is generally well within current capabilities. The real limiting factors concern issues of management, organisation and attitude.

The time is near when it will be appropriate to conduct an experiment on the lines suggested in this scenario. Clearly it is not expected that it would be economically viable in the first instance, however, a tremendous amount would be learned, and it would generate considerable publicity and interest. An international test-bed site could become a proving ground for the introduction and integration of new technology. Such a step is necessary if the industry is to be convinced that this is where its future lies. 


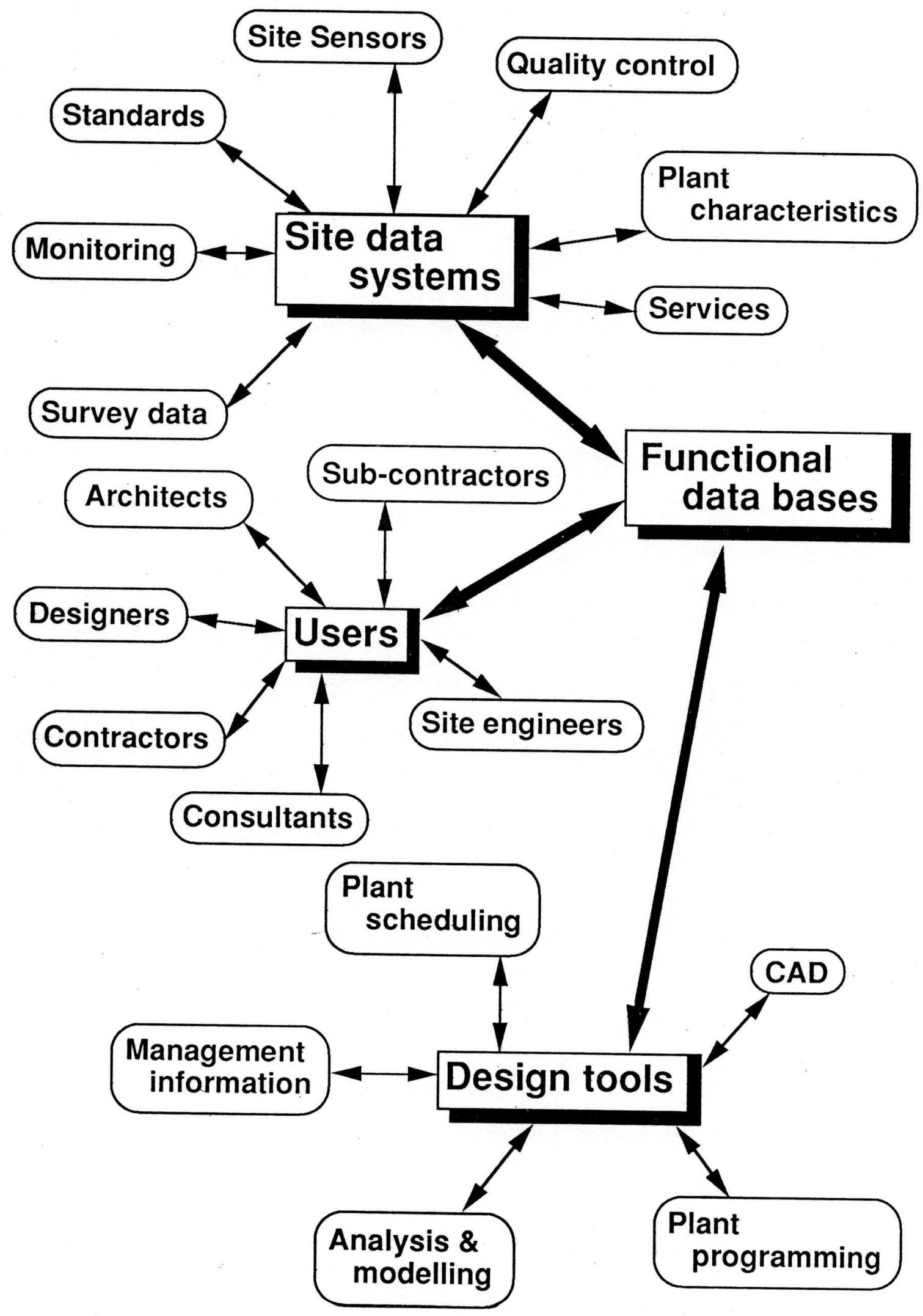

Site 2000 - Information systems and information flows 